\title{
Central Dynamics of Globular Clusters: the Case for a Black Hole in $\omega$ Centauri
}

\author{
Eva Noyola ${ }^{1}$, Karl Gebhardt ${ }^{2}$ and Marcel Bergmann ${ }^{3}$ \\ ${ }^{1}$ Max-Planck-Institut für extraterrestrische Physik, Giessenbachstrasse, 85748, Garching, \\ Germany, email: noyola@mpe.mpg.de \\ ${ }^{2}$ Department of Astronomy, University of Texas, Austin, TX, 78723 \\ ${ }^{3}$ NOAO Gemini Science Center, Casilla 603, La Serena, Chile
}

\begin{abstract}
The globular cluster $\omega$ Centauri is one of the largest and most massive members of the Galactic system. Its classification as a globular cluster has been challenged making it a candidate for being the stripped core of an accreted dwarf galaxy; this and the fact that it has one of the largest velocity dispersions for star clusters in our galaxy makes it an interesting candidate for harboring an intermediate mass black hole. We measure the surface brightness profile from integrated light on an $H S T / \mathrm{ACS}$ image, and find a central power-law cusp of logarithmic slope -0.08. We also analyze Gemini GMOS-IFU kinematic data for a 5" 5 5" field centered on the nucleus of the cluster, as well as for a field 14" away. We detect a clear rise in the velocity dispersion from $18.6 \mathrm{kms}^{-1}$ at 14 " to $23 \mathrm{kms}^{-1}$ in the center. Given the very large core in $\omega$ Cen $\left(2.58^{\prime}\right)$, an increase in the dispersion in the central 10" is difficult to attribute to stellar remnants, since it requires too many dark remnants and the implied configuration would dissolve quickly given the relaxation time in the core. However, the increase could be consistent with the existence of a central black hole. Assuming a constant $M / L$ for the stars within the core, the dispersion profile from these data and data at larger radii implies a black hole mass of $4.0_{-1.0}^{+0.75} \times 10^{4} M_{\odot}$. We have also run flattened, orbit-based models and find a similar mass. In addition, the no black hole case for the orbit model requires an extreme amount of radial anisotropy, which is difficult to preserve given the short relaxation time of the cluster.
\end{abstract}

Keywords. globular clusters: individual (Omega Centauri), Galaxy: kinematics and dynamics

\section{Introduction}

The globular cluster $\omega$ Centauri (NGC 5139) is the most massive member of the Galactic cluster system. It has a measured central velocity dispersion of $22 \pm 4 \mathrm{kms}^{-1}$ (Meylan et al. 1995). A rotating flattened model including proper motion and radial velocity datasets by van de Ven et al. (2006) calculate a total mass of $2.5 \times 10^{6} M_{\odot}$. $\omega$ Cen has a complex stellar population with a broad metallicity distribution (Bedin et al. 2004). These result have led to the hypothesis that $\omega$ Cen is not a classical globular cluster, but instead is the nucleus of an accreted galaxy (Freeman 1993).

Two globular clusters have been suggested for harboring an intermediate mass black hole in their nucleus. One is the galactic cluster M15 (Gerssen et al. 2003) and the other is G1, a giant globular cluster around M31 (Gebhardt, Rich, \& Ho 2005). M15 is assumed to be in a post-core collapse state. Its dynamical state has been debated between harboring a black hole or containing a large number of compact remnants in its center (Baumgardt et al. 2003a). Observational constraints between these two hypothesis remain inconclusive (van den Bosch et al. 2006). G1 on the other hand, has a core with characteristics closer to those of $\omega$ Cen, and observations support the black hole interpretation in this case. Baumgardt et al. (2003b) propose an alternative interpretation for G1 in which they match the observations with a model of two colliding globular clusters. The black hole 


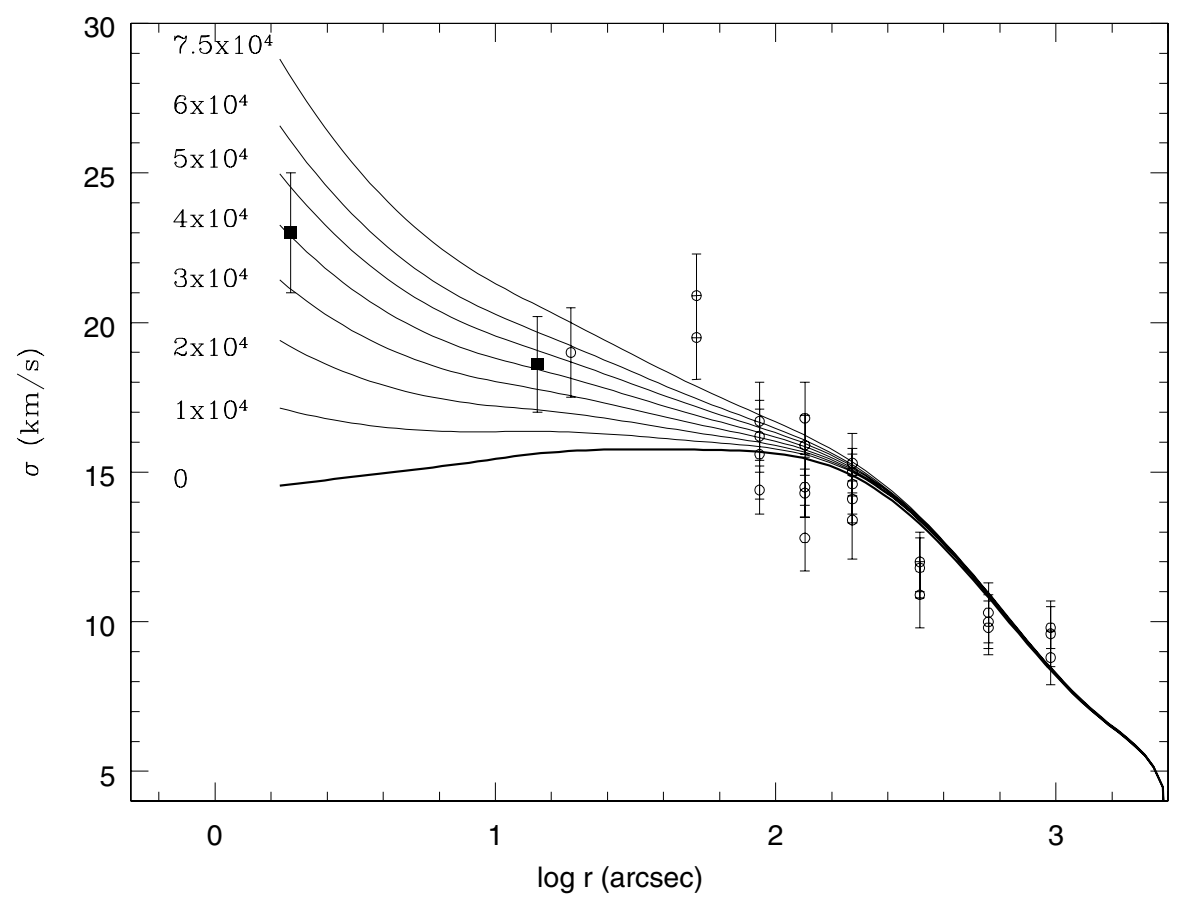

Figure 1. Velocity dispersion profile for $\omega$ Cen with various central black hole models. Filled squares are the dispersions and uncertainties from the GMOS-IFU and open circles are from individual radial velocity measurements. A set of isotropic spherical models of varying black hole masses is shown for comparison. The thick line is the no black hole model and the thin lines represent models with black holes as labeled

interpretation for G1 is strongly supported by radio (Ulvestad et al. 2007) and x-ray (Pooley \& Rappaport 2006) detections centered on the nucleus.

\section{Surface Brightness Profile}

We measure the central part of the surface brightness profile for $\omega$ Cen using $H S T$ spatial resolution. We measure integrated light from an ACS F435W image (340 sec) applying the technique described in detail in Noyola \& Gebhardt (2006). Since the image has a limited radial coverage, we use the Chebychev fit of Trager et al. (1995) for the surface brightness profile to cover the full radial extent of the cluster. The coordinates for our center are RA $13: 26: 46.043$ and DEC $-47: 28: 44.8$ on the ACS dataset J6LP05WEQ using its WCS zeropoint.

The measured surface brightness profile shows a continuous rise toward the center with a logarithmic slope of $-0.08 \pm 0.03$, which is in contrast to the common notion that $\omega$ Cen has a flat core. Baumgardt, Makino \& Hut (2005) perform N-body models of star clusters with initial King profiles and containing a central black hole. They predict the formation of a shallow cusp of -0.1 to -0.3 logarithmic slope after $1.5-4$ relaxation times.

\section{Kinematic Measurements}

We obtained Gemini GMOS-South IFU nod-and-shuffle observations. The IFU has a field of view of $5^{\prime} \times 5^{\prime}$, comprised of 700 individual lenslets plus fiber elements, each of 


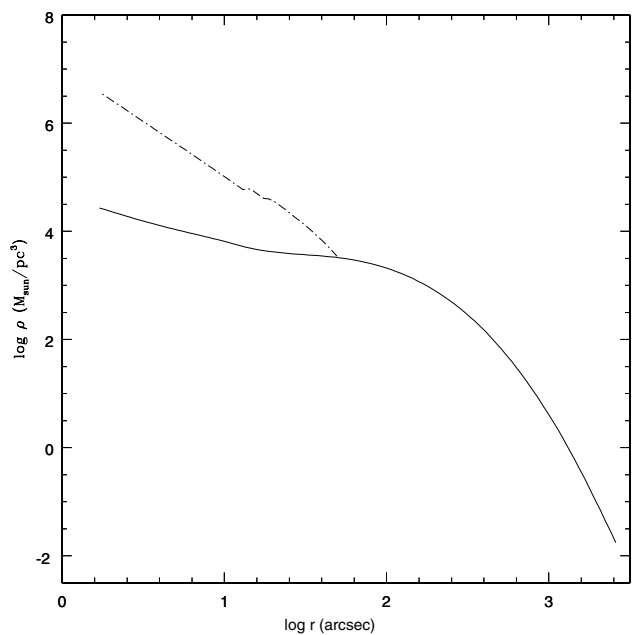

Figure 2. Inferred density profiles. The solid line is the deprojected density profile for the luminous component. The dashed line represents the required dark component to reproduce the observed kinematics.

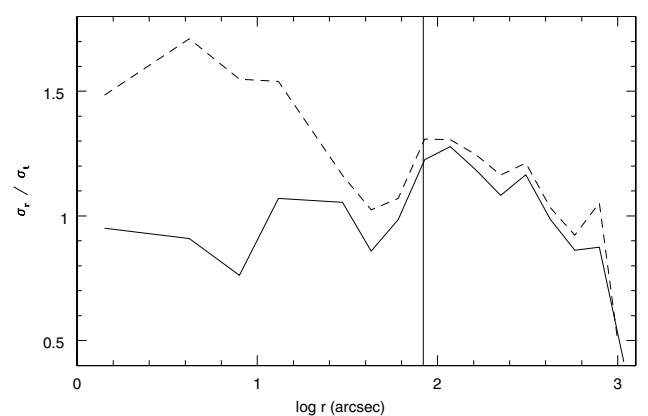

Figure 3. Radial over tangential anisotropy vs. radius from orbit-based models. The solid line is for the best fit model containing a black hole. The dashed line is for a model with no black hole present. The vertical line marks the location of the core radius.

which covers approximately $0^{\prime \prime} .2$ on the sky. We use the R600 grating, yielding a resolving power $\mathrm{R}=5560$, along with the Calcium Triplet filter. Two fields are observed, each for a total integration time of $900 \mathrm{sec}$ on source and $900 \mathrm{sec}$ on sky. The first of the two fields is located at the cluster center, and the second field is centered 14" away. Using the standard tasks from the IRAF-GEMINI package we sky subtract, flat-field, extract the spectra for each fiber, and apply a wavelength calibration.

We match the reconstructed image from the IFU fibers with the same region on the ACS image. Both fields contain $\sim 100$ resolved stars. Using the photometric measurements of individual stars together with the reported seeing, we calculate how many stars contribute to each fiber. Excluding the fibers which are dominated by a single star we estimate that the integrated spectrum of the background unresolved light represent about 60 stars in both fields.

We focus on the Ca triplet region $(8450 \AA-8700 \AA)$ for our analysis. To estimate the velocity dispersion we rely on the integrated light, and require template stars in this case. We use stars observed by Walcher et al. (2005), from VLT-UVES observations at around $\mathrm{R}=35000$. We convolve the spectra to our measured resolving power. To extract the velocity dispersion from the integrated light we utilize the non-parametric, pixel-based technique as described in Gebhardt et al. (2000). We choose an initial velocity profile in bins, convolve it with the set of templates, and calculate residuals to the integrated spectrum. Monte Carlo simulations determine the uncertainties, and use the measured noise in the spectrum. We measure $23.0 \pm 2.0 \mathrm{kms}^{-1}$ for the central field and $18.6 \pm$ $1.6 \mathrm{kms}^{-1}$ for the outer one. Van den Ven et al.(2006) measure a line of sight velocity dispersion profile by combining various datasets. They use 2163 individual radial velocity measurements divided into polar apertures to obtain the final velocity dispersion profile. We use their dispersion estimates. Fig. 1 presents the velocity dispersion data. 


\section{Models}

The presence of an intermediate black hole at the center of this cluster is one of the possibilities for explaining the observed rise in velocity dispersion. We have run two types of modeling in order to test this hypothesis

First, we create a series of models using the non-parametric method described in Gebhardt \& Fischer (1995). We apply a reddening correction to the observed surface brightness profile, which we then deproject using Abel integrals assuming spheroidal symmetry. We apply a spline smoother to the surface brightness profile before deprojecting and thus obtain a luminosity density profile as discussed in Gebhardt et al. (1996). By assuming a mass to light ratio of 2.7 , we calculate a mass density profile, from which the potential and the velocity dispersion can be derived. We repeat the calculation adding a variety of central point masses ranging from 0 to $7.5 \times 10^{4} M_{\odot}$ while keeping the global $M / L$ value fixed. Fig. 1 shows the comparison between the different models and the measured dispersion profile. As it can be seen, an isotropic model with no black hole present predicts a slight decline in velocity dispersion toward the center, instead we observe a clear rise. The predicted central velocity for the no black hole model is $14.6 \mathrm{kms}^{-1}$ which is well below any line of sight velocity dispersion measured inside 1 '. The $\chi^{2}$ curve implies a best-fit black hole mass of $4_{-1}^{+0.75} \times 10^{4} M_{\odot}$.

An alternative to explain the observed rise in $M / L$ toward the center is a concentration of dark stellar remnants. Using the observed velocity dispersion profile, we calculate the total enclosed mass and from this, the mass density profile. We then compare this with the enclosed mass implied by the luminosity density profile, assuming the same M/L as for the isotropic models. We estimate the density profile of the the implied extended dark component, assuming this was the cause of the velocity dispersion rise towards the center. Fig. 2 shows the estimated density profile for the dark and luminous components. If the velocity rise is due to an extended distribution of dark stellar remnants, the density profile of this dark component has to be very concentrated, with a logarithmic slope of $\sim-2.0$, resembling a cluster undergoing core collapse. The relaxation time for $\omega$ Cen implies a much slower dynamical evolution than the one necessary to reach such a configuration. Core-collapse models have shown that when a cluster has reached such a high degree of mass segregation, the observable core to half light radius gets very small, with values below 0.05 (Breeden, Cohn, \& Hut 1994), while this ratio is 0.3 for $\omega$ Cen. Another possibility is that the observed rise in velocity dispersion is due to velocity anisotropy in the cluster. To explore this possibility, we construct orbit-based dynamical models. The models are based on the Schwarzschild formulation and are constructed as in Gebhardt et al. (2000); Gehardt et al. (2003). We use the same deprojection as described above, except we also include the observed flattening. Assuming an $M / L$ ratio and a $\mathrm{BH}$ mass, the mass distribution of the cluster is obtained and from it, the potential can be computed. Using this potential, we generate about $10^{4}$ representative orbits. The best match to the observed photometric and kinematical data provide the orbital weights for a given potential. The process is repeated for various $M / L$ values and $\mathrm{BH}$ masses until the minimum $\chi^{2}$ model is found. The best fit model requires a black hole mass around $3.5 \times 10^{4} M_{\odot}$, close to the isotropic result. As expected, the difference in $\chi^{2}$ compared to the no black hole case is smaller than in the isotropic case. However, the anisotropy profile is extremely different between the black hole and no black hole case. Fig. 3 shows that without the presence of a central black hole, a large degree of radial anisotropy $\left(\sigma_{r} / \sigma_{t}=1.5\right)$ is required inside $0.3 r_{c}$. The models with and without a black hole are close to isotropic for $r>28$ ", which is in agreement with the results of van den Ven et al.(2006). For a system as dense as $\omega$ Cen, such a degree of anisotropy is expected 
to be quickly erased through relaxation processes. Even with this extreme anisotropy profile, the black hole case provides a better match to the data.

\section{Conclusions}

The two pieces of observational evidence that $\omega$ Cen could harbor a central black hole come from the photometry and the kinematics. From the HST image of $\omega$ Cen, we measure a central logarithmic surface brightness slope of $-0.08 \pm 0.03$. This value is very similar to that claimed by the N-body simulations of Baumgardt et al.(2005) that are most likely explained by a central black hole. Standard core-collapse does not lead to such a large core with a shallow central slope. The black hole tends to prevent core collapse while leaving an imprint of a shallow cusp. It will be important to run models tailored to $\omega$ Cen to see if one can cause and maintain a shallow cusp without invoking a central black hole. However, the main observational evidence for the central mass comes from the increase in the central velocity dispersion, where we detect a rise from 18.6 to $23 \mathrm{kms}^{-1}$ from radii of 14 to 2.5 ". In fact, even excluding the Gemini data presented here, the previous ground-based data suggest a central mass concentration as well. The core of $\omega$ Cen is around 155" (about 2.5'), so the dispersion rise is seen well within the core.

\section{References}

Baumgardt, H., Heggie, D. C., Hut, P., \& Makino, J. 2003a, MNRAS, 341, 247

Baumgardt, H., Makino, J., Hut, P., McMillan, S., \& Portegies Zwart, S. 2003b, ApJL, 589, L25

Baumgardt, H., Makino, J., \& Hut, P. 2005, ApJ, 620, 238

Bedin, L., et al. 2004, ApJL, 605, L125

Breeden, J., Cohn, H., \& Hut, P. 1994, ApJ, 421, 195

Freeman, K. C. 1993, in ASP Conf. Ser. 48: The Globular Cluster-Galaxy Connection, 608

Gebhardt, K., \& Fischer, P. 1995, AJ, 109, 209

Gebhardt, K., et al. 1996, AJ, 112, 105

Gebhardt, K., et al. 2000, AJ, 119, 1157

Gebhardt, K., et al. 2003, ApJ, 583, 92

Gebhardt, K., Rich, R. M., \& Ho, L. 2005, ApJ, 634, 1093

Gerssen, J., van der Marel, R., Gebhardt, K., Guhathakurta, P., Peterson, R., Pryor, C. 2003, $A J, 125,376$

Meylan, G., Mayor, M., Duquennoy, A., Dubath, P. 1995, A\&A, 303, 761

Noyola, E., \& Gebhardt, K. 2006, AJ, 132, 447

Pooley, D., \& Rappaport, S. 2006, ApJL, 644, L45

Trager, S., King, I, \& Djorgovski, S. 1995, AJ, 109, 218

Ulvestad et al. 2007, ApJL, 661, L151

van den Bosch et al. 2006, ApJ, 641, 852

van de Ven et al. 2006, A\& $A, 445,513$

Walcher et al. 2005, ApJ, 618, 237 